\title{
Impacto nos atributos do solo sob conversão de floresta para áreas cultivadas na região sul do Amazonas, Brasil
}

\author{
Impact on soil attributes under forest conversion for cultivated \\ areas in the south Amazon Region, Brazil
}

\section{Bruna Firmino Enck'; José Cunegundes Weckner Rodrigues";Abdul Luis Hassane ${ }^{\text {III }}$ Reinato Andrade Tembol ${ }^{\mathrm{V}}$; Milton Cesar Costa Campos ${ }^{\mathrm{V}}$ Luís Antônio Coutrim dos Santos ${ }^{\mathrm{V}}$; Wildson Benedito de Mendes Brito ${ }^{\mathrm{VII}}$}

\section{RESUMO}

A conversão de áreas naturais em agrícola modifica o equilíbrio natural existente, desta forma, à medida que o mesmo é submetido ao uso agrícola alterações negativas ao desenvolvimento vegetal e ao ambiente são observadas. O trabalho teve como objetivo avaliar o impacto nos atributos do solo em ambientes naturais e cultivados no sul do Amazonas. O estudo foi realizado em áreas de floresta nativa, floresta secundária; cultivada com arroz e cultivada com milho em Humaitá, região sul do Amazonas, em cada área foram escolhidos seis pontos aleatórios, totalizando 36 amostras/área. Os solos foram coletados em forma de torrões e anéis volumétricos nas camadas: 0,00-0,10 e 0,10-0,20 $m$, realizou-se análises físicas: densidade do solo (Ds), diâmetro médio geométrico (DMG) e diâmetro médio ponderado (DMP), macroporosidade (MaP), microporosidade (MiP), volume total de poros (VTP) e umidade gravimétrica (Ug); análises químicas: $\mathrm{pH}$ em água, acidez potencial ( $\mathrm{H}+\mathrm{Al})$, alumínio (Al), cálcio (Ca) e magnésio ( $\mathrm{Mg}$ ) trocáveis, carbono orgânico (CO) e estoque de carbono (EC). Posteriormente, os dados foram submetidos às análises de variância e as médias comparadas pelo teste de Tukey. A conversão de floresta nativa para áreas agrícolas imprimiu alterações negativas nos atributos do solo, principalmente na camada superficial de 0,00-0,10 m. Dentre os atributos que apresentaram maior alteração, pode-se destacar a Ds, VTP, DMG, DMP, Ug, CO e EC.

Palavras-chave: Antropização; estoque de carbono; florestas naturais; solos Amazônicos.

\footnotetext{
' Instituto de Educação, Agricultura e Ambiente, Universidade Federal do Amazonas - https://orcid.org/0000-0001-7966-1263 brunaenck@hotmail.com

" Instituto de Educação, Agricultura e Ambiente, Universidade Federal do Amazonas - https://orcid.org/0000-0002-0439-6837 jose_cunegundes@yahoo.com.br

III Instituto de Educação, Agricultura e Ambiente, Universidade Federal do Amazonas - https://orcid.org/0000-0002-9829-5307 assaneluis@gmail.com

IV Instituto de Educação, Agricultura e Ambiente, Universidade Federal do Amazonas - https://orcid.org/0000-0003-3525-9066 reinatoxavier2@gmail.com

v Centro de Ciências Agrárias, Universidade Federal da Paraíba - https://orcid.org/0000-0002-8183-7069 mcesarsolos@gmail.com

VI Universidade do Estado do Amazonas, UEA, Centro de Estudos Superiores de Itacoatiara, CESIT - https://orcid.org/0000-00020824-0901 - santoslac@gmail.com

VIII Instituto de Educação, Agricultura e Ambiente, Universidade Federal do Amazonas- https://orcid.org/0000-0002-4267-5992 wild.brito@gmail.com
} 


\section{ABSTRACT}

The conversion of natural to agricultural areas changes the existing natural balance, thus, as it is subjected to agricultural use, negative changes to plant development and the environment are observed. The work aimed to evaluate the impact on soil attributes in natural and cultivated environments in southern Amazonas state. The study was carried out in areas: native forest, secondary forest; cultivated with rice and cultivated with corn in Humaitá, southern Amazonas, in each area six random points were chosen, totaling 36 samples / area.The soils were collected in the form of clumps and volumetric rings in the layers: $0.00-0.10$ and $0.10-0.20 \mathrm{~m}$, physical analyzes were performed: bulk density (Bd), geometric mean diameter (GMD) and weighted average diameter (WAD), macroporosity $(\mathrm{MaP})$, microporosity (MiP), total pore volume (TPV) and gravimetric moisture (Gm); chemical analysis: $\mathrm{pH}$ in water, potential acidity ( $\mathrm{H}+\mathrm{Al})$, aluminum (Al), exchangeable calcium (Ca) and magnesium (Mg), organic carbon (OC) and carbon stock (CS). Subsequently, the data were subjected to analysis of variance and the means compared by the Tukey test. The conversion of native forest to agricultural areas led to negative changes in soil attributes, especially in the $0.00-0.10 \mathrm{~m}$ surface layer. Among the attributes that showed the greatest change, the Bd, TPV, GMD, WAD, Gm, OC and CS can be highlighted.

Keywords: Amazonian soils; anthropization carbon stock; natural forests.

\section{INTRODUÇÃO}

O solo é um componente de suma importância do ecossistema terrestre, pois, além de ser o principal substrato utilizado pelas plantas para o seu desenvolvimento, exerce ainda uma multiplicidade de funções como: distribuição, regulação e infiltração da água, armazenamento e ciclagem de nutrientes essenciais e outros elementos, além de exercer ação filtrante e protetora da qualidade da água e do ar (BRADY, 2013).

Na região Amazônica os solos normalmente apresentam grandes limitações químicas, devido sua baixa disponibilidade de nutrientes e capacidade de troca de cátions (CUNHA et al., 2007), portanto, a fertilidade do solo neste ambiente tem sua manutenção fortemente associada ao equilíbrio entre a cobertura vegetal e os processos biogeoquímicos (MOLINE; COUTINHO, 2015), estando diretamente ligada a ciclagem de nutrientes contidos na matéria orgânica do solo (MOREIRA; MALAVOLTA, 2004; CARDOSO et al., 2009).

Por outro lado, o solo quando mantido em condições naturais, sob vegetação nativa e em equilíbrio, normalmente apresenta características físicas (densidade do solo, porosidade, permeabilidade e estrutura) adequadas ao crescimento normal das 
plantas (ANDREOLA et al., 2000). Todavia, a conversão de áreas naturais em agrícola modifica o equilíbrio natural existente, alterando as propriedades do solo (MULLER et al., 2001). Portanto, a perturbação antrópica do sistema solo-cobertura vegetal natural, geralmente causa perdas de carbono, implicando à redução do seu teor ao longo do tempo e a degradação da qualidade do solo no desempenho das suas funções básicas (DORAN; PARKIN, 1994).

Neste contexto, a quantificação de alterações nos atributos químicos e físicos do solo é um fator primordial que tem sido utilizado para monitorar a qualidade dos solos (ZANINETTI et al., 2016). Como esses atributos são sensíveis e diretamente influenciados pelas práticas de manejo do solo, é de suma importância a sua quantificação, para então estabelecer medidas de manejo que visem a obtenção de sistemas agrícolas sustentáveis baseado na qualidade de cada solo (CUNHA NETO et al., 2018).

Atualmente, a conversão e ocupação de áreas antes florestadas por áreas agrícolas sem o devido critério técnico e um dos principais problemas causados pela ação antrópica na região Amazônica, principalmente na região conhecida como arco do desmatamento (CAMPOS et al., 2015). Problemas estes que afetam diretamente a preservação dos recursos naturais, e consequentemente, as propriedades físicas e químicas dos solos nos diversos ambientes. Neste sentido, a compreensão e a quantificação dos impactos dos diferentes sistemas de manejo nas propriedades físicas e químicas do solo, são de fundamentais importâncias para a conservação desse recurso natural (CUNHA NETO et al., 2018).

Dentro deste cenário, a maioria dos estudos na região sul do Amazonas têm demostrado ampla diversidade de resultados, com variação entre os locais estudados com ou sem continuidade espacial, provavelmente associada ao sistema de manejo e as características intrínsecas dos solos (CAMPOS, 2012; CAMPOS et al., 2015; FREITAS et al., 2017). Portanto, torna-se necessário realizar estudos avaliando as propriedades físicas e químicas dos solos em áreas naturais e manejadas na região Amazônica que sofreram substituição da vegetação natural para implantação de cultivos agrícolas, 
para se entender as possíveis variações que ocorrem no solo devido às ações antrópicas (CAMPOS et al., 2015). Diante do exposto, o presente trabalho teve como objetivo avaliar os atributos físicos e químicos do solo em ambientes naturais e cultivados em Humaitá, sul do Amazonas.

\section{MATERIAL E MÉTODOS}

O estudo foi conduzido em Humaitá, região sul do Amazonas, estando situada de acordo com a classificação de Köppen no clima tropical chuvoso (chuvas do tipo monção), com curto período seco, apresentando início das chuvas em outubro e prolongando-se até junho. A temperatura média anual da região varia de 25 a $27^{\circ} \mathrm{C}$ e precipitação média anual de 2.500 mm com umidade relativa do ar entre 85 a 90\% (BRASIL, 1978). A vegetação típica e em maior escala nas proximidades é constituída por árvores adensadas e multiestratificadas entre 20 e 30 m de altura.

Com a finalidade de avaliar atributos físicos e químicos dos solos em áreas naturais e cultivadas, foram selecionadas quatro áreas na região sul do Amazonas, sendo uma área natural (floresta nativa), uma floresta secundária e duas cultivadas (arroz e milho) (Figura 1). De acordo com Campos (2009) todas as áreas avaliadas estão sob Cambissolo Háplico Alítico plíntico.

As áreas selecionadas apresentam as características conforme a seguir:

a) Área 1 - Floresta nativa: área com floresta em condição natural, sob coordenadas geográfica: $7^{\circ} 36^{\prime} 54.5^{\prime \prime} \mathrm{S}$ e $63^{\circ}$ 05' $25.8^{\prime \prime}$, caracterizada como sem intervenção humana;

b) Área 2 - Floresta secundária: área com floresta secundária sob coordenadas $7^{\circ}$ 31' 40.9" S e $63^{\circ} 03^{\prime} 15^{\prime \prime}$ W, caracterizada pela derrubada da vegetação no passado pela ação humana, atualmente em processo de recuperação florestal;

b) Área 3 - Cultivo de arroz: área cultivada com arroz nos últimos anos, sob coordenadas $7^{\circ} 37^{\prime} 00.3^{\prime \prime} \mathrm{S}$ e $63^{\circ} 05^{\prime} 34.2^{\prime \prime} \mathrm{W}$, a área ficou em pousio, sendo cultivada há dois anos com o arroz; 
4) Área 4 - Cultivo de milho: área cultivada com arroz nos últimos quatro anos com coordenadas $7^{\circ} 36^{\prime} 53.5^{\prime \prime} \mathrm{S}$ e $63^{\circ}$ 05' 09.4" W, cultivada incialmente com soja, pousio, pastagem e agora retornando com a soja e as safrinhas de milho. Durante o preparo da área foi incorporado calcário para correção da acidez do solo.

Figura 1 - Mapa de localização das áreas naturais e cultivadas em Humaitá, sul do Amazonas

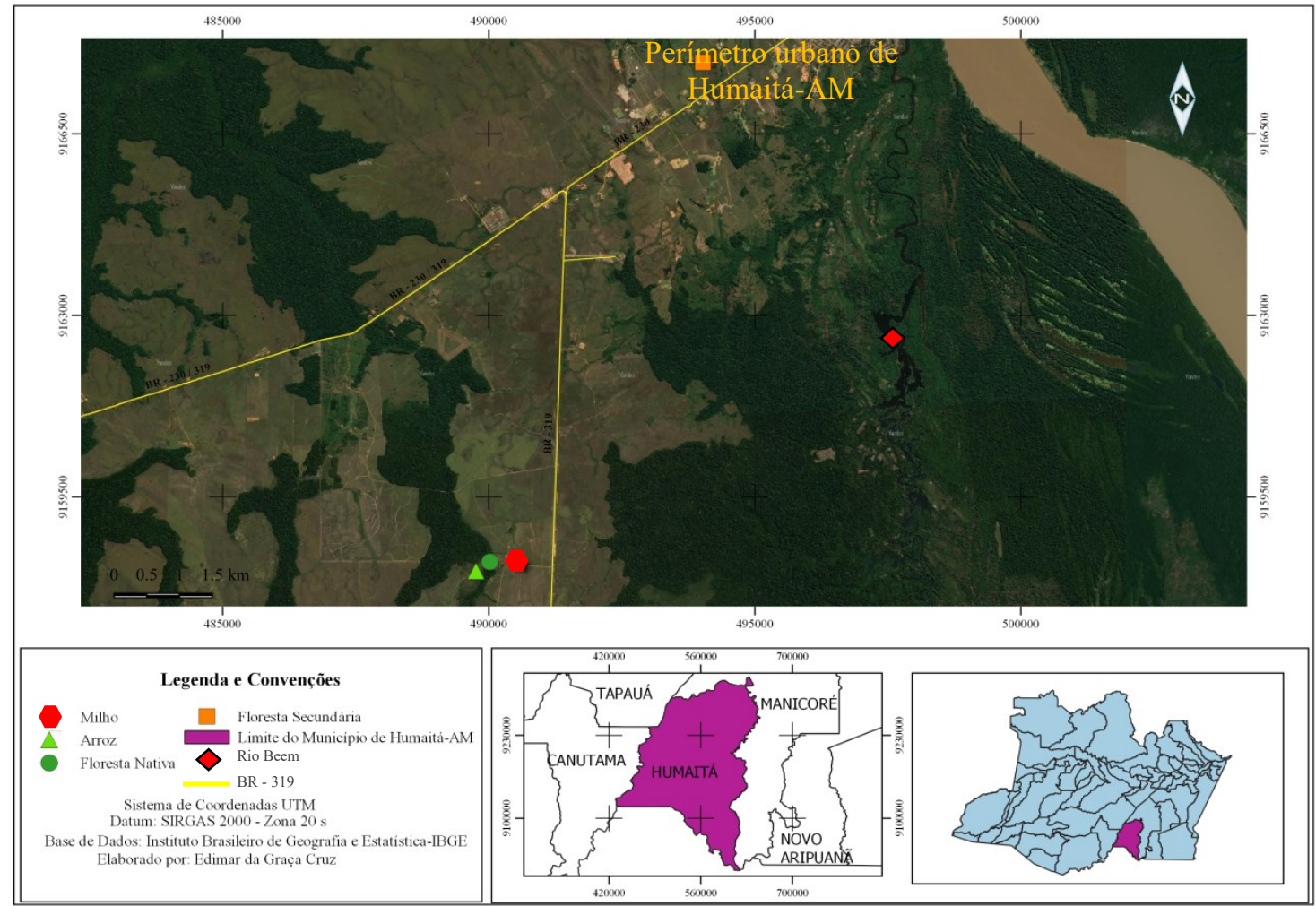

Fonte: ENCK, 2019

As áreas de estudo foram georreferenciadas com auxílio de um equipamento de GPS modelo GPSmap 62S, marca GARMIM, com precisão de 5 m, sistema operacional UTM - WGS 84, em cada área foram coletados 6 pontos distribuídos aleatoriamente. Em cada ponto foram coletadas duas amostras em anel volumétrico e uma amostra em forma de torrão em cada camada de 0,00-0,10 e 0,10-0,20 m.

As amostras coletadas foram secas à sombra e levemente destorroadas de forma manual, posteriormente foram passadas em um jogo de peneiras de diferentes malhas, a primeira peneira de 9,51 mm de diâmetro de malha, a segunda peneira de 
4,76 mm de diâmetro, onde o material retido nela foi utilizado para determinação da estabilidade de agregação do solo. Já para obtenção da terra fina seca ao ar (TFSA) para realização das análises físicas e químicas do solo foram passadas em peneira de $2,00 \mathrm{~mm}$ de malha.

A determinação dos agregados foi realizado, segundo o método de Kemper; Chepil (1965), com modificações nas seguintes classes de diâmetro: 4,76-2,0 mm; 2,01,0 mm; 1,0-0,50 mm; 0,50-0,25 mm;0,25-0,125; 0,125-0,063 mm. Os agregados foram colocados em contato com a água sobre a peneira de $2,0 \mathrm{~mm}$ e submetidos à agitação vertical em aparelho Yoder por 15 min e com 32 oscilações por minuto. 0 material retido em cada classe das peneiras foi colocado em estufa a $105^{\circ} \mathrm{C}$, e em seguida mensurada as respectivas massas em uma balança digital.

Os resultados foram expressos em estabilidade dos agregados avaliados pelo diâmetro médio ponderado (DMP), obtido pela fórmula proposta por Castro Filho et al. (1998), e o diâmetro médio geométrico (DMG) segundo Schaller; Stockinger (1953).

Nas determinações da densidade do solo (Ds), macroporosidade (MaP) e microporosidade (MiP), volume total de poros (VTP) e umidade gravimétrica (Ug), as amostras coletadas em anéis volumétricos foram saturadas por meio da elevação gradual, até dois terços da altura do anel, de uma lâmina de água numa bandeja plástica. Após a saturação, as amostras foram pesadas e levadas à mesa de tensão para determinação da MiP do solo, sendo submetidas a uma tensão de $-0,006 \mathrm{MPa}$ (DONAGEMA et al., 2011).

Em relação às determinações químicas, foram determinados: pH em água na relação solo: água de 1:2,5; acidez potencial $(\mathrm{H}++\mathrm{Al} 3+)$ foi determinada pelo método do acetato de cálcio 0,5 mol L-1 tamponado a $\mathrm{pH} 7,0$ sendo titulado com $\mathrm{NaOH}$ em presença de fenolftaleína como indicador; alumínio (Al3+), cálcio (Ca2+) e magnésio (Mg2+) trocáveis foram extraídos com a solução extratora de $\mathrm{KCl} 1 \mathrm{~mol} \mathrm{~L}-1$. O alumínio foi determinado utilizando $\mathrm{NaOH} \mathrm{0,025} \mathrm{mol} \mathrm{L-1} \mathrm{como} \mathrm{titulante} \mathrm{em} \mathrm{presença}$ de azul de bromotimol como indicador colorimétrico. Cálcio e magnésio foram determinados por espectrofotometria de absorção atômica, todas as análises foram 
realizadas de acordo com Donagema et al. (2011). O carbono orgânico foi determinado pelo método da oxidação via úmida, com aquecimento externo (WALKLEY; BLACK, 1934; YEOMANS; BREMNER, 1988). Já o estoque de carbono (EC) foi definido pela equação:

$\mathrm{EC}=(\mathrm{Ds} \times \mathrm{h} \times \mathrm{CO}) / 10$

em que:

$\mathrm{EC}=$ estoque de carbono (Mg ha-1);

Ds = densidade do solo $(\mathrm{g} \mathrm{cm}-3)$;

$\mathrm{h}=\mathrm{a}$ espessura da camada de solo amostrada (cm);

$\mathrm{CO}=$ teor de $\mathrm{CO}(\mathrm{g} . \mathrm{kg}-1)$.

Para avaliação dos dados foram empregados o uso de análises estatísticas descritivas, sendo calculadas: média, mediana, mínimo, máximo, desvio padrão, coeficiente de variação, assimetria e curtose. A hipótese de normalidade dos dados foi averiguada pelo teste de normalidade de Kolmogorov-Smirnov a 5\% de probabilidade. Para comparação das médias afim de diferenciar os ambientes, foi utilizado o teste de Tukey a 5\% de probabilidade. As análises foram realizadas no programa estatístico IBM SPSS Statistics 21.0 (IBM Corp., 2012).

\section{RESULTADOS E DISCUSSÃO}

Os resultados da estatística descritiva e da análise de variância dos atributos físicos estudados em áreas naturais e cultivadas são apresentados nas Tabela 1 e 2, para as camadas 0,00-0,10 e 0,10-0,20 m, respectivamente. Como pode ser observado nos resultados os valores de média e mediana apresentam valores muito próximos, com exceção para o atributo MaP, da área sob cultivo de milho. 
Tabela 1 - Estatística descritiva e análise de variância dos atributos físicos avaliados em áreas naturais e cultivadas na camada 0,00-0,10 m em Humaitá, sul do Amazonas

\begin{tabular}{|c|c|c|c|c|c|c|c|}
\hline \multirow{2}{*}{$\begin{array}{l}\text { Estatística } \\
\text { Descritiva }^{1}\end{array}$} & Ds & MaP & MiP & VTP & DMG & DMP & Ug \\
\hline & $\mathrm{g} \mathrm{cm}^{-3}$ & $\mathrm{~m}^{3} \mathrm{~m}^{-3}$ & & & $\mathrm{~mm}$ & & $\mathrm{MPa}$ \\
\hline \multicolumn{8}{|c|}{ Área sob cultivo de Arroz } \\
\hline Média & $1,17 \mathbf{a}$ & $13,90 \mathbf{a}$ & $32,11 \mathbf{a b}$ & $46,01 \mathbf{a b}$ & 2,03 bc & 2,65 bc & $27,45 \mathrm{c}$ \\
\hline Mediana & 1,15 & 11,33 & 31,96 & 44,12 & 2,05 & 2,73 & 26,98 \\
\hline Mínimo & 1,06 & 6,26 & 27,82 & 38,11 & 1,29 & 2,10 & 25,62 \\
\hline Máximo & 1,29 & 28,06 & 37,77 & 61,38 & 2,68 & 3,04 & 29,96 \\
\hline DP & 0,09 & 8,38 & 3,46 & 8,65 & 0,57 & 0,37 & 1,64 \\
\hline CV (\%) & 7,89 & 60,25 & 10,78 & 18,79 & 28,15 & 13,81 & 5,96 \\
\hline Assimetria & 0,38 & 1,10 & 0,65 & 1,28 & $-0,13$ & $-0,61$ & 0,72 \\
\hline Curtose & $-1,33$ & 0,39 & 0,55 & 1,53 & $-1,74$ & $-1,06$ & $-0,73$ \\
\hline K-S & $0,25 *$ & 0,21 * & $0,20 *$ & 0,21 * & $0,19 *$ & $0,19 *$ & $0,23 *$ \\
\hline \multicolumn{8}{|c|}{ Área sob cultivo de Milho } \\
\hline Média & $1,37 \mathbf{a}$ & $8,56 \mathbf{a}$ & $30,22 \mathbf{b}$ & $38,79 \mathbf{b}$ & $1,65 \mathrm{c}$ & $2,49 \mathrm{c}$ & $22,26 \mathrm{c}$ \\
\hline Mediana & 1,36 & 5,80 & 30,34 & 35,81 & 1,61 & 2,47 & 22,27 \\
\hline Mínimo & 1,20 & 1,76 & 28,19 & 32,07 & 0,95 & 1,94 & 19,47 \\
\hline Máximo & 1,56 & 23,95 & 32,22 & 53,12 & 2,59 & 3,09 & 24,91 \\
\hline DP & 0,12 & 8,16 & 1,41 & 8,05 & 0,65 & 0,44 & 2,13 \\
\hline CV (\%) & 8,87 & 95,27 & 4,67 & 20,75 & 39,38 & 17,82 & 9,56 \\
\hline Assimetria & 0,43 & 1,73 & $-0,11$ & 1,40 & 0,35 & 0,17 & $-0,06$ \\
\hline Curtose & 0,40 & 3,10 & $-0,18$ & 1,44 & $-1,38$ & $-1,46$ & $-1,58$ \\
\hline $\mathrm{K}-\mathrm{S}$ & $0,19 *$ & $0,25 *$ & $0,13 *$ & $0,24^{*}$ & $0,17 *$ & $0,14^{*}$ & $0,15^{*}$ \\
\hline \multicolumn{8}{|c|}{ Área sob Floresta Secundária } \\
\hline Média & $0,96 \mathbf{b}$ & $17,05 \mathbf{a}$ & $31,45 \mathbf{a b}$ & $48,5 \mathbf{a b}$ & $2,75 \mathbf{a}$ & $3,17 \mathbf{a}$ & $33,06 \mathbf{b}$ \\
\hline Mediana & 1,00 & 16,60 & 34,14 & 48,70 & 2,76 & 3,16 & 32,66 \\
\hline Mínimo & 0,65 & 11,09 & 24,89 & 38,90 & 2,47 & 3,07 & 27,60 \\
\hline Máximo & 1,12 & 24,55 & 35,13 & 55,06 & 2,99 & 3,25 & 38,27 \\
\hline DP & 0,17 & 4,94 & 4,94 & 5,78 & 0,18 & 0,06 & 3,65 \\
\hline CV (\%) & 17,79 & 28,94 & 15,72 & 11,91 & 6,40 & 1,89 & 11,04 \\
\hline Assimetria & $-1,41$ & 0,44 & $-0,91$ & $-0,76$ & $-0,33$ & $-0,25$ & $-0,05$ \\
\hline Curtose & 2,03 & $-0,71$ & $-1,87$ & 0,66 & 0,68 & 0,69 & 0,37 \\
\hline $\mathrm{K}-\mathrm{S}$ & $0,33 *$ & $0,20 *$ & $0,33 *$ & $0,18 *$ & $0,15 *$ & $0,23 *$ & 0,20 * \\
\hline \multicolumn{8}{|c|}{ Área sob Floresta Nativa } \\
\hline Média & $0,85 \mathbf{b}$ & $15,55 \mathbf{a}$ & 36,03 a & $51,58 \mathbf{a}$ & $2,82 \mathbf{a}$ & $3,10 \mathbf{a b}$ & $42,61 \mathbf{a}$ \\
\hline Mediana & 0,84 & 14,42 & 36,19 & 51,45 & 2,95 & 3,19 & 42,45 \\
\hline Mínimo & 0,73 & 10,13 & 31,96 & 46,67 & 1,96 & 2,62 & 36,19 \\
\hline Máximo & 1,01 & 22,32 & 38,76 & 57,18 & 3,32 & 3,23 & 47,62 \\
\hline DP & 0,10 & 5,40 & 2,47 & 3,64 & 0,46 & 0,24 & 4,58 \\
\hline CV $(\%)$ & 11,19 & 34,73 & 6,86 & 7,05 & 16,34 & 7,62 & 10,74 \\
\hline Assimetria & 0,71 & 0,43 & $-0,76$ & 0,32 & $-1,54$ & $-2,42$ & $-0,24$ \\
\hline Curtose & 0,82 & $-2,00$ & 0,45 & 0,26 & 3,32 & 5,89 & $-1,72$ \\
\hline $\mathrm{K}-\mathrm{S}$ & $0,15 *$ & 0,21 * & $0,17 *$ & $0,18 *$ & $0,28 *$ & $0,46 *$ & 0,21 * \\
\hline
\end{tabular}

'DP: Desvio padrão; CV\%: coeficiente de variação, \%; K-S: teste de normalidade Kolmogorov-Smirnov; *significativo à 5\%; Ds: densidade do solo; MaP: macroporos; MiP: microporos; VPT: volume total de poros; DMG: diâmetro médio geométrico; DMP: diâmetro médio ponderado; UG.: umidade gravimétrica. Médias seguidas por letras diferentes, na mesma coluna, são significativamente diferentes ao nível de 5\% pelo teste de Tukey 
Tabela 2 - Estatística descritiva e análise de variância dos atributos físicos avaliados em áreas naturais e cultivadas na camada 0,10-0,20 m em Humaitá, sul do Amazonas

\begin{tabular}{|c|c|c|c|c|c|c|c|}
\hline \multirow{2}{*}{$\begin{array}{l}\text { Estatística } \\
\text { Descritiva }^{1}\end{array}$} & Ds & $\mathrm{MaP}$ & MiP & VTP & DMG & DMP & Ug \\
\hline & $\mathrm{g} \mathrm{cm}^{-3}$ & $\mathrm{~m}^{3} \mathrm{~m}^{-3}$ & & & $\mathrm{~mm}$ & & $\mathrm{MPa}$ \\
\hline \multicolumn{8}{|c|}{ Área sob cultivo de Arroz } \\
\hline Média & $1,32 \mathbf{a b}$ & $6,78 \mathbf{a}$ & $32,78 \mathbf{a b}$ & $39,86 \mathbf{a}$ & $1,54 \mathbf{a}$ & $2,12 \mathbf{a}$ & 25,15 bc \\
\hline Mediana & 1,31 & 7,08 & 33,17 & 40,19 & 1,61 & 2,22 & 24,78 \\
\hline Mínimo & 1,21 & 4,52 & 29,74 & 36,42 & 0,83 & 1,25 & 22,54 \\
\hline Máximo & 1,43 & 7,84 & 36,46 & 43,30 & 2,24 & 2,75 & 27,98 \\
\hline DP & 0,07 & 1,19 & 2,47 & 2,59 & 0,51 & 0,57 & 2,36 \\
\hline CV (\%) & 5,27 & 17,49 & 7,54 & 6,50 & 33,16 & 26,59 & 9,39 \\
\hline Assimetria & 0,22 & $-1,78$ & 0,17 & $-0,07$ & $-0,15$ & $-0,61$ & 0,22 \\
\hline Curtose & 2,13 & 3,59 & $-0,47$ & $-1,37$ & $-0,74$ & $-0,74$ & $-2,40$ \\
\hline K-S & $0,17^{*}$ & $0,30 *$ & $0,26 *$ & $0,21 *$ & $0,14^{*}$ & $0,18^{*}$ & $0,24^{*}$ \\
\hline \multicolumn{8}{|c|}{ Área sob cultivo de Milho } \\
\hline Média & $1,41 \mathbf{a}$ & $11,92 \mathbf{a}$ & $31,3 \mathbf{b}$ & 40,59 a & $1,78 \mathbf{a}$ & $2,51 \mathbf{a}$ & $21,67 \mathrm{c}$ \\
\hline Mediana & 1,42 & 12,33 & 31,46 & 39,39 & 1,83 & 2,65 & 22,45 \\
\hline Mínimo & 1,22 & 3,49 & 30,13 & 27,44 & 1,10 & 1,94 & 16,57 \\
\hline Máximo & 1,55 & 21,46 & 32,43 & 53,50 & 2,33 & 2,95 & 24,69 \\
\hline DP & 0,12 & 6,95 & 0,93 & 9,64 & 0,52 & 0,45 & 2,95 \\
\hline CV (\%) & 8,22 & 58,28 & 2,97 & 23,75 & 29,02 & 17,71 & 13,62 \\
\hline Assimetria & $-0,74$ & 0,04 & $-0,29$ & 0,05 & $-0,29$ & $-0,60$ & $-1,15$ \\
\hline Curtose & 0,67 & $-1,23$ & $-1,70$ & $-1,09$ & $-1,70$ & $-1,93$ & 1,02 \\
\hline K-S & 0,20 * & $0,18^{*}$ & $0,19 *$ & $0,19 *$ & $0,18 *$ & 0,21 * & $0,25 *$ \\
\hline \multicolumn{8}{|c|}{ Área sob Floresta Secundária } \\
\hline Média & $1,18 \mathbf{b}$ & $5,61 a$ & $32,93 \mathbf{a b}$ & $38,54 \mathbf{a}$ & $2,31 \mathbf{a}$ & $2,88 \mathbf{a}$ & $28,11 \mathbf{b}$ \\
\hline Mediana & 1,19 & 6,06 & 32,97 & 39,00 & 2,33 & 3,02 & 26,54 \\
\hline Mínimo & 0,99 & 1,85 & 30,49 & 36,43 & 1,40 & 2,12 & 24,96 \\
\hline Máximo & 1,29 & 7,67 & 34,78 & 40,91 & 2,97 & 3,25 & 34,97 \\
\hline DP & 0,11 & 2,15 & 1,68 & 1,71 & 0,54 & 0,41 & 3,86 \\
\hline CV (\%) & 9,31 & 38,41 & 5,11 & 4,45 & 23,54 & 14,12 & 13,73 \\
\hline Assimetria & $-1,08$ & $-1,17$ & $-0,30$ & $-0,14$ & $-0,78$ & $-1,67$ & 1,41 \\
\hline Curtose & 1,20 & 1,20 & $-1,10$ & $-0,97$ & 0,92 & 3,00 & 1,36 \\
\hline K-S & $0,18^{*}$ & $0,23 *$ & $0,22 *$ & $0,25 *$ & $0,22 *$ & $0,26 *$ & $0,26 *$ \\
\hline \multicolumn{8}{|c|}{ Área sob Floresta Nativa } \\
\hline Média & $0,96 \mathbf{c}$ & $12,96 \mathbf{a}$ & $35,03 \mathbf{a}$ & 47,99 a & $2,14 \mathbf{a}$ & $2,63 \mathbf{a}$ & $36,94 \mathbf{a}$ \\
\hline Mediana & 0,98 & 11,41 & 35,10 & 47,38 & 1,85 & 2,65 & 35,80 \\
\hline Mínimo & 0,78 & 4,83 & 31,79 & 41,96 & 0,96 & 2,02 & 32,56 \\
\hline Máximo & 1,09 & 24,54 & 37,88 & 56,32 & 3,82 & 3,15 & 42,37 \\
\hline DP & 0,12 & 7,70 & 2,48 & 5,87 & 1,15 & 0,48 & 3,89 \\
\hline CV (\%) & 12,60 & 59,42 & 7,09 & 12,23 & 53,65 & 18,38 & 10,54 \\
\hline Assimetria & $-0,58$ & 0,60 & $-0,14$ & 0,37 & 0,62 & $-0,13$ & 0,60 \\
\hline Curtose & $-1,05$ & $-1,11$ & $-2,25$ & $-1,81$ & $-1,35$ & $-2,33$ & $-1,44$ \\
\hline $\mathrm{K}-\mathrm{S}$ & $0,24 *$ & $0,20 *$ & 0,20 * & $0,23 *$ & $0,19 *$ & 0,20 * & $0,27 *$ \\
\hline
\end{tabular}

1DP: Desvio padrão; CV\%: coeficiente de variação, \%; K-S: teste de normalidade Kolmogorov-Smirnov; *significativo à 5\%; Ds: densidade do solo; MaP: macroporos; MiP: microporos; VPT: volume total de poros; DMG: diâmetro médio geométrico; DMP: diâmetro médio ponderado; UG.: umidade gravimétrica. Médias seguidas por letras diferentes, na mesma coluna, são significativamente diferentes ao nível de 5\% pelo teste de Tukey. 
Os coeficientes de assimetria e curtose dos atributos físicos estudados apresentaram valores próximos à zero para maioria dos atributos, exceções foram observadas para os atributos MaP sob cultivo de milho, Ds sob floresta secundária, DMG e DMP sob floresta nativa na camada de 0,00-0,10 m (Tabela 1), enquanto que na camada de 0,10-0,20 m os atributos MaP sob cultivo de arroz, Ds, MaP e DMP sob floresta secundária foram os atributos que apresentaram valores de curtose e assimetria mais distantes de zero (Tabela 2), resultados com valores semelhantes para os atributos físicos do solo também foram observados por Kamimura et al. (2013). Trabalhos como o de Cortez et al. (2011) e Alho et al. (2016) destacam que a assimetria e curtose são indicadores da distribuição dos dados e valores próximos a zero indicam maior normalidade dos dados.

Os resultados do teste de Kolmogorov-Smirnov (K-S) mostram que todos os atributos físicos avaliados apresentam normalidade, em ambas as camadas estudadas (Tabela 1 e 2) justificando os valores encontrados para média e mediana, os quais indicavam uma normalidade dos dados em razão da proximidade dos valores centrais observados para cada atributo.

Todas as variáveis apresentam coeficiente de variação (CV\%) variando de baixo a médio, com exceção do atributo MaP das áreas sob cultivo de arroz e milho na camada de 0,00-0,10 m (Tabela 1) que apresenta alta variação, de acordo com os critérios propostos por Warrick; Nielsen (1980), que classificam os valores do CV\% abaixo de $12 \%$ como baixa, entre $12 \%$ e $60 \%$ como média e valores acima $60 \%$ como alta variação. Estes resultados corroboram com Aquino et al. (2015) e Alho et al. (2016), que também verificaram variação baixa à média para os atributos físicos em trabalhos avaliando solos da região Sul do Amazonas.

Os resultados da análise de variância mostram que as áreas cultivadas com milho e arroz apresentam estatisticamente maior densidade do solo (1,37 e 1,17 $\mathrm{g} \mathrm{cm}^{-}$ 3 , respectivamente) quando comparadas com as áreas de floresta natural e floresta secundária $\left(<1 \mathrm{~g} \mathrm{~cm}^{-3}\right)$, por meio do teste de Tukey $(\mathrm{p}<0,05)$ (Tabela 1). Resultado semelhante aos observados por Freitas et al. (2018), que estudando os valores de 
estoque de carbono de Latossolos em sistemas de manejo natural e alterado; verificaram maiores valores de Ds em ambientes antropizados. Estes maiores resultados são justificados pela compactação causada pelo tráfego de máquinas e implementos agrícolas utilizados durante as operações de preparo, semeadura e colheita na área (CAVENAGE et al., 1999; HAMZA \& ANDERSON, 2005; VIANA et al., 2011), assim como, com o tempo de exploração dessas áreas (SOUZA et al., 2019).

Os maiores valores de Ds nas áreas cultivadas também estão relacionadas com a perda de carbono pela conversão de áreas nativas em agrícolas, corroborando Viana et al. (2011) que atribuem o aumento da Ds em áreas cultivadas à redução dos teores de matéria orgânica, em comparação com o solo sob mata nativa. Valores estatisticamente superiores de carbono nas áreas sob vegetação nativa poderão ser confirmados neste trabalho na Tabela 3. Os valores para a segunda camada 0,10-0,20 m, foram semelhantes a primeira camada $(0,00-0,10 \mathrm{~m})$, onde o menor valor de Ds foi observado para o solo de floresta nativa $\left(0,96 \mathrm{~g} \mathrm{~cm}^{-3}\right)$ e o maior para área cultivada com milho $\left(1,41 \mathrm{~g} \mathrm{~cm}^{-3}\right)$ (Tabela 2$)$.

Os valores de Ds das áreas cultivadas estão acima dos valores médios considerados ideais para Ds, os quais segundo Camargo; Alleoni (1997) estão situados na faixa de 1,0 e 1,2 $\mathrm{g} \mathrm{cm}^{-3}$, podendo resultar assim, em uma maior degradação, indicando uma possível compactação e/ou adensamento do solo na área de estudo. Segundo Dexter (2004), o aumento da Ds geralmente provoca a diminuição da porosidade, tamanho e continuidade de poros. Streck et al. (2004) e Freddi et al. (2007) também constataram que não só a porosidade e continuidade de poros diminuem com o aumento da Ds, mas também, a disponibilidade de nutrientes e água às plantas, além de provocar alterações na morfologia do sistema radicular com conseguinte redução da produtividade da cultura.

Em relação aos atributos MaP, MiP e VPT é possível verificar que a MaP em ambas as camadas (Tabela 1 e 2) e o VTP na camada de 0,10-0,20 m não apresentaram diferença significativa pelo teste Tukey a nível de $5 \%$ de probabilidade, sendo esta observada apenas para os atributos MiP em ambas as camadas e VTP na 
camada de 0,00-0,10 m. Em relação aos valores de MaP, vale ressaltar que com exceção da área sob cultivo de milho na camada de 0,00-0,10 m e das áreas sob floresta secundária e cultivo de arroz na camada de 0,10-0,20 m, todas as demais áreas em ambas as camadas apresentam valores superiores a $10 \mathrm{~m}^{3} \mathrm{~m}^{-3}$, o qual é definido como limitante às condições favoráveis ao crescimento radicular e desenvolvimento das plantas (DEXTER, 1988). Fato importante, uma vez que, os macroporos são a principal rota do crescimento das raízes (CAMARGO; ALLEONI, 1997).

Os maiores valores de MiP em ambas camadas e VTP na camada 0,00-0,10 m, foram encontradas na área sob floresta nativa, já os menores, foram observados na área sob cultivo de milho, e valores intermediários foram observados para as áreas de floresta secundária e arroz (Tabela 1 e 2). Maiores resultados de VTP em áreas naturais quando comparado com áreas cultivadas também foi observado por Luciano et al. (2010) estudando propriedades físicas e carbono orgânico do solo sob plantio direto comparados à mata natural. Vale ressaltar ainda, que Ds e VTP apresentam uma relação inversa, desta forma, os maiores valores de VTP observados na área de floresta nativa deste trabalho está relacionada com os menores valores de Ds da mesma.

Analisando os valores médios dos índices de estabilidade de agregado DMG e DMP observou-se que os maiores valores estatisticamente significativos pelo teste de Tukey $(p<0,05)$ foram encontrados nas áreas naturais, em comparação com as áreas antropizadas (Tabela 1). Verifica-se também, que o solo sob cultivo de milho apresenta os menores valores de DMG e DMP, evidenciando maior degradação na estrutura do mesmo. Provavelmente os maiores valores observados nas áreas sob florestas são justificados pelo maior aporte de material vegetal nestas áreas, o qual contribui para o aumento dos teores de matéria orgânica e, consequentemente, aumento da agregação do solo (KATO et al. 2010; MANTOVANELLI et al., 2015). Ainda de acordo com Mantovanelli et al. (2015) o manejo realizado nas áreas cultivadas influencia diretamente a formação, estruturação e estabilização dos agregados. 
A área de floresta nativa apresentou os maiores valores de Ug, seguida pela área sob floresta seminativa, que apresentou valores intermediários e pelas $x^{\prime} x^{\prime} x^{\prime}$ áreas cultivadas com milho e arroz que apresentaram os menores valores para o atributo, em ambas as camadas. Os resultados de Ug encontrados neste trabalho corroboram com Reichert et al. (2007), onde os autores afirmam que em solos sob áreas florestais os mesmos apresentam uma tendência de concentrar e manter a umidade do solo estável devido ao sombreamento e ao maior acúmulo de serrapilheira proveniente das árvores.

Os resultados da estatística descritiva e análise de variância dos atributos químicos do solo avaliados em áreas naturais e cultivadas nas camadas de 0,00-0,10 e 0,10-0,20 m são apresentados nas Tabelas 3 e 4, respectivamente. Como pode ser observado, as medidas de tendência central, média e mediana dos atributos químicos, mostram valores muito próximos, justificando uma distribuição normal ou aproximadamente normal dos dados, confirmados pelos coeficientes de assimetria e curtose que apresentaram valores próximos à zero para maioria dos atributos.

O resultado do teste de K-S confirma a normalidade dos atributos químicos em ambas as camadas (Tabela 3 e 4). Quanto ao CV\%, os atributos químicos do solo apresentam baixa à media variabilidade para todos os atributos analisados, as únicas exceções foram observadas para os atributos $\mathrm{Al}^{3+}, \mathrm{Mg}^{2+}$ e EC que apresentaram alta variabilidade de acordo com a classificação proposta por Warrick; Nielsen (1980).

No que se refere aos resultados da análise de variância dos atributos químicos, por meio do teste de Tukey $(p<0,05)$, pode-se observar que todos os atributos foram estatisticamente significativos (Tabela 3 e 4), evidenciando que existe diferença entre os ambientes naturais e cultivados. Os valores de pH em água são extremamente ácidos para todos os solos e profundidades avaliadas. Estes resultados são característicos dos solos da região sul do Amazonas em que os solos normalmente apresentam valores de $\mathrm{pH}$ em água inferiores a 5,00 (CAMPOS et al., 2012a; MANTOVANELLI et al., 2015 e AQUINO et al., 2016). 
Tabela 3 - Estatística descritiva e análise de variância dos atributos químicos avaliados em áreas naturais e cultivadas na camada de 0,00-0,10 m em Humaitá, sul do Amazonas

\begin{tabular}{|c|c|c|c|c|c|c|c|}
\hline \multirow{2}{*}{$\begin{array}{l}\text { Estatística } \\
\text { Descritiva }^{1}\end{array}$} & \multirow{2}{*}{$\mathrm{pH}$} & $\mathrm{Al}$ & $\mathrm{Al}+\mathrm{H}$ & $\mathrm{Ca}^{2+}$ & $\mathrm{Mg}^{2+}$ & $\mathrm{CO}$ & EC \\
\hline & & \multicolumn{4}{|c|}{------------'cmolc.kg-1'----------- } & g.kg-1 & $\mathrm{Mg} \mathrm{ha}^{-1}$ \\
\hline \multicolumn{8}{|c|}{ Área sob cultivo de Arroz } \\
\hline Média & $3,97 \mathbf{b}$ & $2,92 \mathbf{b}$ & $17,90 \mathbf{b}$ & $0,79 b$ & $0,47 a$ & 19,48 c & $22,66 \mathbf{b}$ \\
\hline Mediana & 3,94 & 2,96 & 18,07 & 0,79 & 0,52 & 19,71 & 22,56 \\
\hline Mínimo & 3,70 & 2,70 & 16,67 & 0,94 & 0,64 & 14,55 & 18,84 \\
\hline Máximo & 4,29 & 3,10 & 18,81 & 0,63 & 0,18 & 24,12 & 27,68 \\
\hline DP & 0,25 & 0,15 & 0,71 & 0,11 & 0,16 & 3,07 & 3,27 \\
\hline CV (\%) & 6,35 & 5,03 & 3,99 & 13,62 & 33,92 & 15,77 & 14,42 \\
\hline Assimetria & 0,26 & $-0,52$ & $-0,93$ & $-0,17$ & $-1,52$ & $-0,21$ & 0,45 \\
\hline Curtose & $-2,14$ & $-0,73$ & 1,95 & 0,43 & 2,83 & 2,06 & $-0,47$ \\
\hline K-S & $0,19 *$ & 0,21 * & $0,21 *$ & $0,05^{*}$ & $0,07 *$ & $0,25 *$ & $0,15^{\star}$ \\
\hline \multicolumn{8}{|c|}{ Área sob cultivo de Milho } \\
\hline Média & $4,99 \mathbf{a}$ & $0,81 \mathbf{c}$ & $8,08 \mathrm{c}$ & $1,17 a$ & $0,54 a$ & $20,77 \mathrm{c}$ & $28,46 \mathbf{a b}$ \\
\hline Mediana & 5,02 & 0,90 & 8,09 & 0,55 & 0,14 & 20,95 & 30,40 \\
\hline Mínimo & 4,60 & 0,50 & 6,60 & 0,56 & 0,27 & 16,79 & 20,23 \\
\hline Máximo & 5,22 & 1,10 & 9,90 & 0,50 & 0,04 & 24,25 & 32,72 \\
\hline $\mathrm{DP}$ & 0,22 & 0,26 & 1,15 & 0,02 & 0,08 & 2,56 & 4,69 \\
\hline CV (\%) & 4,33 & 31,38 & 14,26 & 4,59 & 54,75 & 12,35 & 16,50 \\
\hline Assimetria & $-1,25$ & $-0,60$ & 0,44 & $-1,46$ & 0,51 & $-0,35$ & $-1,34$ \\
\hline Curtose & 2,03 & $-1,74$ & 0,20 & 1,67 & 1,03 & 0,38 & 1,09 \\
\hline K-S & $0,27 *$ & $0,29 *$ & $0,17 *$ & 0,10 * & $0,05 *$ & $0,19 *$ & $0,29 *$ \\
\hline \multicolumn{8}{|c|}{ Área sob Floresta Secundária } \\
\hline Média & $3,98 \mathbf{b}$ & $5,78 \mathbf{a}$ & $23,81 \mathbf{a}$ & $0,54 c$ & $0,14 b$ & $31,25 \mathbf{b}$ & $29,50 \mathbf{a b}$ \\
\hline Mediana & 3,98 & 5,65 & 23,51 & 1,17 & 0,47 & 29,72 & 29,44 \\
\hline Mínimo & 3,91 & 5,00 & 21,78 & 1,32 & 0,80 & 27,36 & 25,48 \\
\hline Máximo & 4,04 & 6,80 & 25,91 & 1,04 & 0,33 & 39,17 & 35,88 \\
\hline DP & 0,05 & 0,62 & 1,79 & 0,09 & 0,19 & 4,51 & 3,75 \\
\hline CV (\%) & 1,15 & 10,68 & 7,53 & 8,01 & 35,99 & 14,44 & 12,71 \\
\hline Assimetria & $-0,23$ & 0,71 & 0,29 & 0,39 & 0,65 & 1,28 & 0,92 \\
\hline Curtose & $-0,21$ & 0,80 & $-2,09$ & 0,51 & $-1,74$ & 1,12 & 0,93 \\
\hline K-S & $0,10 *$ & $0,18^{*}$ & 0,21 * & $0,08^{*}$ & $0,14^{*}$ & $0,23 *$ & $0,23 *$ \\
\hline \multicolumn{8}{|c|}{ Área sob Floresta Nativa } \\
\hline Média & $3,85 \mathbf{b}$ & $3,55 \mathbf{b}$ & $16,20 \mathbf{b}$ & $0,55 c$ & $0,05 b$ & $38,56 \mathbf{a}$ & $32,98 \mathbf{a}$ \\
\hline Mediana & 3,89 & 3,45 & 15,76 & 0,55 & 0,06 & 38,52 & 32,33 \\
\hline Mínimo & 3,54 & 3,00 & 13,86 & 0,57 & 0,06 & 29,16 & 23,69 \\
\hline Máximo & 4,11 & 4,30 & 19,63 & 0,54 & 0,03 & 44,96 & 45,40 \\
\hline DP & 0,25 & 0,49 & 2,48 & 0,01 & 0,01 & 5,67 & 7,03 \\
\hline CV (\%) & 6,50 & 13,66 & 15,29 & 2,05 & 25,80 & 14,70 & 21,32 \\
\hline Assimetria & $-0,21$ & 0,63 & 0,40 & 0,13 & $-1,13$ & $-0,72$ & 0,95 \\
\hline Curtose & $-2,60$ & $-0,65$ & $-2,01$ & $-2,51$ & 0,73 & 0,67 & 2,69 \\
\hline K-S & $0,28 *$ & $0,20 *$ & $0,25 *$ & $0,07 *$ & $0,11^{*}$ & $0,19 *$ & 0,30 * \\
\hline
\end{tabular}

1DP: Desvio padrão; CV\%: coeficiente de variação, \%; K-S: teste de normalidade Kolmogorov-Smirnov; *significativo à 5\%; Médias seguidas por letras diferentes, na mesma coluna, são significativamente diferentes ao nível de 5\% pelo teste de Tukey. 
Tabela 4 - Estatística descritiva e análise de variância dos atributos químicos avaliados em áreas naturais e cultivadas na camada de 0,10-0,20 m em Humaitá, sul do Amazonas

\begin{tabular}{|c|c|c|c|c|c|c|c|}
\hline \multirow{2}{*}{$\begin{array}{l}\text { Estatística } \\
\text { Descritiva }^{1}\end{array}$} & \multirow{2}{*}{$\mathrm{pH}$} & $\mathrm{Al}$ & $\mathrm{Al}+\mathrm{H}$ & $\mathrm{Ca}^{2+}$ & $\mathrm{Mg}^{2+}$ & $\mathrm{CO}$ & EC \\
\hline & & \multicolumn{4}{|c|}{ 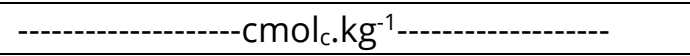 } & g.kg-1 & $\mathrm{Mg} \mathrm{ha}^{-1}$ \\
\hline \multicolumn{8}{|c|}{ Área sob cultivo de Arroz } \\
\hline Média & $3,71 \mathrm{c}$ & $4,82 a$ & $19,96 \mathbf{a}$ & $0,60 b$ & $0,22 b$ & $15,40 \mathbf{b}$ & $20,17 \mathbf{b}$ \\
\hline Mediana & 3,75 & 4,90 & 20,13 & 0,60 & 0,52 & 14,24 & 19,64 \\
\hline Mínimo & 3,31 & 3,80 & 18,48 & 0,67 & 0,64 & 11,69 & 15,29 \\
\hline Máximo & 3,95 & 5,40 & 20,96 & 0,54 & 0,18 & 20,15 & 24,43 \\
\hline DP & 0,23 & 0,55 & 0,87 & 0,05 & 0,16 & 3,26 & 3,57 \\
\hline CV (\%) & 6,17 & 11,33 & 4,37 & 8,07 & 33,92 & 21,17 & 56,56 \\
\hline Assimetria & $-1,10$ & $-1,52$ & $-0,95$ & 0,39 & $-1,52$ & 0,64 & 0,07 \\
\hline Curtose & 1,22 & 3,16 & 0,92 & $-0,53$ & 2,83 & $-1,19$ & $-1,18$ \\
\hline K-S & $0,17 *$ & $0,32 *$ & $0,21 *$ & $0,18^{*}$ & $0,07 *$ & $0,23 *$ & $0,20 *$ \\
\hline \multicolumn{8}{|c|}{ Área sob cultivo de Milho } \\
\hline Média & $4,63 \mathbf{a}$ & $1,23 \mathrm{c}$ & $11,60 \mathrm{c}$ & $0,98 a$ & $0,42 a$ & $16,58 \mathbf{a b}$ & $23,41 \mathbf{a b}$ \\
\hline Mediana & 4,63 & 1,00 & 9,90 & 0,99 & 0,47 & 16,35 & 22,32 \\
\hline Mínimo & 4,40 & 0,40 & 8,75 & 1,15 & 0,80 & 14,05 & 20,18 \\
\hline Máximo & 4,93 & 2,90 & 16,50 & 0,82 & 0,33 & 20,15 & 30,41 \\
\hline $\mathrm{DP}$ & 0,18 & 0,92 & 3,52 & 0,11 & 0,19 & 2,07 & 3,61 \\
\hline CV (\%) & 3,81 & 74,96 & 30,30 & 11,32 & 35,99 & 12,48 & 15,42 \\
\hline Assimetria & 0,72 & 1,39 & 0,91 & 0,13 & 0,65 & 0,92 & 0,84 \\
\hline Curtose & 1,61 & 1,91 & $-1,74$ & 0,40 & $-1,74$ & 1,58 & 1,74 \\
\hline K-S & $0,23^{*}$ & $0,22 *$ & $0,34 *$ & $0,05^{*}$ & $0,13^{*}$ & $0,20 *$ & $0,32 *$ \\
\hline \multicolumn{8}{|c|}{ Área sob Floresta Secundária } \\
\hline Média & $4,06 \mathbf{b}$ & $5,47 \mathbf{a}$ & $18,10 \mathbf{a b}$ & $0,55 b$ & $0,09 b c$ & $23,31 \mathbf{a b}$ & $27,14 \mathbf{a}$ \\
\hline Mediana & 4,06 & 5,65 & 17,98 & 0,55 & 0,14 & 21,45 & 29,95 \\
\hline Mínimo & 3,90 & 4,30 & 13,70 & 0,57 & 0,27 & 18,23 & 22,27 \\
\hline Máximo & 4,16 & 6,50 & 24,59 & 0,54 & 0,04 & 33,78 & 33,50 \\
\hline $\mathrm{DP}$ & 0,09 & 0,81 & 4,23 & 0,01 & 0,08 & 5,73 & 4,27 \\
\hline CV (\%) & 2,31 & 14,76 & 23,38 & 2,70 & 54,75 & 24,60 & 15,73 \\
\hline Assimetria & $-0,94$ & $-0,35$ & 0,51 & $-0,11$ & 0,51 & 1,50 & 0,40 \\
\hline Curtose & 1,36 & $-0,86$ & $-0,86$ & $-2,73$ & 1,03 & 2,17 & $-1,12$ \\
\hline $\mathrm{K}-\mathrm{S}$ & $0,28 *$ & $0,20 *$ & $0,18 *$ & $0,09 *$ & $0,15^{*}$ & $0,22 *$ & $0,19 *$ \\
\hline \multicolumn{8}{|c|}{ Área sob Floresta Nativa } \\
\hline Média & 4,01 bc & $3,17 \mathbf{b}$ & 14,08 bc & $0,54 b$ & $0,03 c$ & $24,69 a$ & $22,97 \mathbf{a b}$ \\
\hline Mediana & 4,03 & 3,15 & 14,11 & 0,54 & 0,06 & 25,46 & 24,35 \\
\hline Mínimo & 3,62 & 2,80 & 12,05 & 0,56 & 0,06 & 13,97 & 15,22 \\
\hline Máximo & 4,30 & 3,50 & 16,50 & 0,51 & 0,03 & 35,85 & 27,80 \\
\hline DP & 0,26 & 0,31 & 1,70 & 0,02 & 0,01 & 7,62 & 4,96 \\
\hline CV (\%) & 6,46 & 9,72 & 12,10 & 3,76 & 25,80 & 30,88 & 21,59 \\
\hline Assimetria & $-0,47$ & 0,04 & 0,17 & $-0,20$ & $-1,13$ & 0,01 & $-0,70$ \\
\hline Curtose & $-0,79$ & $-2,43$ & $-1,07$ & $-1,53$ & 0,73 & $-0,02$ & $-0,83$ \\
\hline K-S & $0,16 *$ & $0,21 *$ & $0,18^{*}$ & $0,11 *$ & $0,05^{*}$ & $0,17 *$ & $0,23 *$ \\
\hline
\end{tabular}


Os baixos valores de pH em água encontrados são em decorrência da alta precipitação da região amazônica que causa lixiviação das bases trocáveis da camada superficial para o restante do perfil do solo (CRAVO et al., 2012), consequentemente, o aumento da concentração de íons $\mathrm{H}^{+}$no solo (REIS et al., 2009), o qual é provocada pelo processo de intemperismo influenciado pelas altas temperaturas e longos períodos de precipitação.

Os maiores valores de $\mathrm{pH}$ em água foram observados para a área sobre cultivo de milho 4,99 e 4,63 nas camadas de 0,00-0,10 e 0,10-0,20 m, respectivamente. Estes valores são justificados pelo uso da calagem durante o preparo da área para o cultivo de milho, prática utilizada como corretivo da acidez do solo por meio da adição de carbonato de cálcio e magnésio ao solo, feita normalmente na camada de 0,00-0,20 m do solo, com necessidades de reavaliação e novas aplicações após cinco anos (NICOLODI et al., 2008).

Em relação aos valores de alumínio trocável observou-se que os maiores valores foram encontrados na área sob floresta secundária em ambas as profundidades (Tabela 3 e 4). Valores altos de alumínio são característicos dos solos da região (CAMPOS et al., 2012b; SANTOS et al., 2012).

A acidez potencial do solo $\left(\mathrm{H}^{+}+\mathrm{Al}^{3+}\right)$ apresentaram a mesma tendência dos teores de $\mathrm{Al} 3+$, exibindo os menores valores para a área sob cultivo de milho e os maiores teores para as áreas nativas, em ambas as profundidades (Tabela 3 e 4). Estes resultados também são decorrentes da prática de calagem aplicadas durante o preparo da área para o cultivo de milho. De acordo com Mantovanelli et al. (2015), a diminuição do Al trocável em ambientes manejados decorre, principalmente, dos efeitos da calagem, os quais contribuem para a neutralização desse elemento no solo.

Os efeitos da calagem foram responsáveis pelo aumento do $\mathrm{pH}$ em água, diminuição dos teores de $\mathrm{Al}^{3+}$ e $\mathrm{H}^{+}+\mathrm{Al}^{3+}$ e, além disso, proporcionou o aumento significativo dos teores de cálcio e magnésio no solo sob cultivo de milho, corroborando assim com Amarantes et al. (2007), que avaliando a influência da calagem no acúmulo de nutrientes observaram um aumento significativo dos valores 
de $\mathrm{pH}$, cálcio e magnésio. Os baixos teores de cálcio e magnésio encontrados nos solos naturais, são resultantes das altas precipitações na região Amazônica, baixa capacidade de retenção de nutrientes e troca de cátions, devido principalmente, ao domínio de mineralogia oxídica e caulinítica, bem como, dos baixos valores de carbono orgânico no solo (LEHMANN et al., 2003).

Nas camadas de 0,00-0,10 e 0,10-0,20 m, os teores de $\mathrm{Ca}^{2+}$ foram maiores na área sob o cultivo de milho diferindo estatisticamente das demais áreas, fato explicado pela pratica de calagem realizada recentemente. Em relação aos teores de $\mathrm{Mg}^{2+}$ verifica-se que na camada de 0,00-0,10 m estes são maiores nas áreas cultivadas quando comparadas as áreas de floresta nativa e secundaria diferindo estatisticamente, para a camada 0,10-0,20 m também houve diferenças significativas entre as áreas, com teores mais altos na área sob cultivo do milho > sob cultivo de arroz > floresta secundária > floresta nativa.

Em relação aos teores de carbono orgânico, os maiores valores foram observados para a área de floresta nativa, apresentando valores estatisticamente diferente quando comparados com a área sob cultivo de arroz, e não diferindo estatisticamente das áreas de floresta secundária e sob cultivo de milho. Os maiores valores observados para a área de floresta nativa são decorrentes do maior aporte de resíduos vegetais e da não perturbação do sistema (LOSS et al., 2015; MANTOVANELLI et al., 2015). Neste contexto, Moline \& Coutinho (2015), relatam que a conservação de áreas naturais para agrícolas na Amazônia acarreta em significativa redução de matéria orgânica depositada nas camadas superficiais do solo, resultando em alterações negativas na disponibilidade de nutrientes, o qual juntamente com o manejo inadequado do solo, diminui a capacidade produtiva das áreas agrícolas da região.

No que se refere aos valores de EC o maior teor foi observado para o solo sob floresta nativa, sendo os menores verificados para o solo sob cultivo de arroz, enquanto o solo sob floresta secundária e milho, apresentaram valores intermediários (Tabela 3), corroborando assim com Freitas et al. (2018), que 
estudando o estoque de carbono de Latossolos em sistemas de manejo natural e alterado, encontraram maior EC para a área de mata nativa. Desta forma, os processos de antropização através de práticas agrícolas reduz o EC no solo a níveis inferiores ao encontrado em condições de floresta nativa (CALONEGO et al., 2012; FREITAS et al., 2018).

\section{CONCLUSÃO}

A conversão de floresta nativa para áreas agrícolas imprimiu alterações negativas nos atributos físicos e químicos do solo, principalmente na camada superficial de 0 - 10 cm. Dentre os atributos que apresentaram maior alteração, podemos destacar a densidade do solo, porosidade total, os índices de agregados diâmetro médio geométrico e ponderado, umidade, carbono orgânico e estoque de carbono.

Os atributos de acidez do solo e das bases trocáveis apresentaram melhores resultados para a área cultivada com milho, sendo estes resultados decorrentes dos efeitos da prática de calagem realizada durante o preparo da área. Estes atributos são extremamente limitantes em áreas naturais do Amazonas, portanto, a prática da calagem é uma obrigação para uma boa produtividade agrícola.

\section{REFERÊNCIAS}

ALHO, L. C.; CAMPOS, M. C. C.; MANTOVANELLI, B. C.; SILVA, D. M. P.; SOUZA, Z. M.; CUNHA, J. M.; SOARES, M. D. R. Physical and geospatial attributes of inceptisols and ultisols under native vegetation in Humaitá, AM, Brazil. Bioscience Journal, v. 32, n. 2, p. 422-430, 2016.

AMARANTE, C.V.T; ERNANI, P.R.; SOUZA, A.G. Influência da calagem e da adubação fosfatada no acúmulo de nutrientes e crescimento da erva de São João. Horticultura Brasileira, v.25, p.533-537, 2007

ANDREOLA, F.; COSTA, L. M.; OLSZEVSKI, N. Influência da cobertura vegetal de inverno e da adubação orgânica e, ou, mineral sobre as propriedades físicas de uma Terra Roxa Estruturada. Revista Brasileira de Ciência do Solo, v.24, p.857-865, 2000. 
AQUINO, R. E. De; CAMPOS, M. C. C.; SOARES, M. D. R.; OlIVEIRA, I. A. De; FRANCISCON, U.; SILVA, D. M. P. Da; CUNHA, J. M. Da. Chemical soil attributes evaluated by multivariate techniques and geostatistics in the area with agroforestry and sugarcane in Humaitá, AM, Brazil. Bioscience Journal, v. 32, n. 1, p. 61-72, 2016.

AQUINO, R. E.; CAMPOS, M. C. C.; JUNIOR, J. M.; OLIVEIRA, I. A.; TEIXEIRA, D. B.; CUNHA, J. M. Use of scaled semivariograms in the planning sample of soil physical properties in southern Amazonas, Brazil. Revista Brasileira de Ciência do Solo, v. 39, n. 2, p. 21-30, 2015.

BRADY, N. C. Elemento da natureza e propriedade do solo. Ciências do solo. $3^{a}$ Edição tradução técnica: Igo Fernando Lopsch, Porto Alegre Bookman. p, 186, 2013.

BRASIL. Ministério das Minas e Energia. Projeto Radam Brasil. Rio de Janeiro: EMBRAPA. p. 561, 1978.

BRASIL. Ministério das Minas e Energia. Projeto Radambrasil - Folha SB. 20, Purus. Rio de Janeiro, 1978. $561 \mathrm{p}$.

CALONEGO, J.C.; SANTOS, C.H.; TIRITAN, C.S.; CUNHA JÚNIOR, J.R. Estoque de carbono e propriedades físicas de solos submetidos a diferentes sistemas de manejo. Revista Caatinga, v. 24, p. 128-35, 2012.

CAMARGO, O.A.; ALLEONI, L.R.F. Compactação do solo e o desenvolvimento das plantas. Piracicaba: USP/ESALQ, 1997.132p.

CAMPOS, M. C. C. Pedogeomorfologia aplicada à ambientes amazônicos do médio Rio Madeira, 2009. 242 p. Tese (Doutorado em Ciências do Solo) - Universidade Federal Rural de Pernambuco. Departamento de Agronomia.

CAMPOS, M. C. C.; RIBEIRO, M. R. SOUZA JUNIOR, V. S.; RIBEIRO FILHO, M. R.; ALMEIDA, M. C. Topossequência de solos na transição Campos naturais-floresta na região de Humaitá, Amazonas. Acta Amazônica, v. 42, n.3, p. 387 - 398, 2012b.

CAMPOS, M. C. C.; RIBEIRO, M. R.; SOUZA JÚNIOR, V.S.; RIBEIRO FILHO, M. R.; OLIVEIRA, I. A. Interferências dos pedoambientes nos atributos do solo em uma topossequência de transição Campos/Floresta. Revista Ciência Agronômica, v.41, p.527-535, 2010.

CAMPOS, M. C. C.; SANTOS, L. A. C. dos; SILVA, D. M. P. da; MANTOVANELLI, B. C.; SOARES, M. D. R. Caracterização física e química de terras pretas arqueológicas e de solos não antropogênicos na região de Manicoré, Amazonas. Revista Agro@mbiente, v. 6, n. 2, p.102-109, 2012a.

CAMPOS, M. C. C.; BERGAMIN, A. C.; CUNHA, J. M.; OLIVEIRA, I. A. Caracterização dos atributos do solo em ambiente natural e transformado na região Sul do Amazonas. Goiânia: Editora da PUC-GOIÁS, v. 1000, p. 218, 2015. 
CAMPOS, M. C.C Caracterização e gênese de solos em diferentes ambientes fisiograficos na região sul do Amazonas. Goiânia: Ed. Da PUC Goiás, 112, 2012.

CASTRO FILHO, C.; MUZILLI, O.; PODANOSCHI, A. L. Estabilidade dos agregados e sua relação com o teor de carbono orgânico em um Latossolo Roxo Distrófico, em função de sistemas de plantio, rotações de culturas e métodos de preparo das amostras. Revista Brasileira de Ciência do Solo, v. 22, n. 3, p.527-538, 1998.

CAVENAGE, A. et al. Alterações nas propriedades físicas de um Latossolo VermelhoEscuro sob diferentes culturas. Revista Brasileira de Ciência do Solo, v. 23, n. 4, p. 997-1003, 1999.

CORTEZ, J. W.; ALVES, A. D. S.; MOURA, M. R. D.; OLSZEVSKI, N.; NAGAHAMA, H. J. Atributos físicos do Argissolo Amarelo do semiárido nordestino sob sistemas de preparo. Revista Brasileira de Ciência do Solo, v. 35, n. 3, p. 1207-1216, 2011.

CRAVO, M. S.; SMYTH, T. J.; BRASIL, E. C. Calagem em latossolo amarelo distrófico da Amazônia e sua influência em atributos químicos do solo e na produtividade de culturas anuais. Revista Brasileira de Ciência do Solo, v. 36, n. 3, p. 895-908, 2012.

CUNHA NETO, F. V., PEREIRA, M. G., SANTOS LELES, P. S., \& SILVA ABEL, E. L. Atributos Químicos e Físicos do Solo em Áreas sob Diferentes Coberturas Florestais e Pastagem em Além Paraíba - MG. Revista Ciência Florestal, v. 28, n.1, p.13-24, 2018.

CUNHA, T. J. F.; MADARI, B. E.; BENITES, V. M.; CANELLAS, L. P.; NOVOTNY, E. H.; MOUTTA, R. O.; TROMPOWSKY, P.; SANTOS, G. A. Fracionamento químico da matéria orgânica e características de ácidos húmicos de solos com horizonte a antrópico da Amazônia (Terra Preta). Acta Amazônica, v. 37, p. 91-98, 2007.

DEXTER, A. R. Advances in characterization of soil structure. Soil \& Tillage Research, v. 11, n.3, p. 199-238, 1988.

DEXTER, A. R. Soil physical quality. Part I. Theory, effects of soil texture, density, and organic matter, and effects on root growth. Geoderma, v. 120, p. 201-214, 2004.

DONAGEMA, G. K.; CAMPOS, D. V. B. de; CALDERANO, S. B.; TEIXEIRA, W. G.; VIANA, J. H. M. (Org.). Manual de métodos de análise do solo. 2.ed. rev. Rio de Janeiro: Embrapa Solos (Embrapa Solos. Documentos, 132), p. 230. 2011.

DORAN, J. W.; PARKIN, T. B. Defining and assessing soil quality. In: DORAN, J. W. etal. (Eds.) Defining soil quality for a sustainable environment. Madisson: ASA/SSSA, p. 3-21, 1994.

FREDDI, O. D. S.; CENTURION, J. F.; BEUTLER, A. N.; ARATANI, R. G.; LEONEL, C. L. Compactação do solo no crescimento radicular e produtividade da cultura do milho.

Revista Brasileira de Ciência do Solo, v. 31, n. 4, p. 627-636, 2007. 
FREITAS, L.; OlIVEIRA, I. A.; CASAGRANDE, J. C.; SIlVA, L. S.; CAMPOS, M. C. C. Estoque de carbono de latossolos em sistemas de manejo natural e alterado. Ciência Florestal, v. 28, n. 1, p. 228-239, 2018.

FREITAS, L.; OLIVEIRA, I. A.; SILVA, L. S.; FRARE, J. C. V.; FILLA, V. A.; GOMES, R. P. Indicadores da qualidade química e física do solo sob diferentes sistemas de manejo. Revista Unimar Ciências. p.18-28, 2017.

HAMZA, M. A.; ANDERSON, W. K. Soil compaction in cropping systems: a review of the nature, causes and possible solutions. Soil \& Tillage Research, v. 82, n. 2, p. 121-145, 2005.

IBM Corp. Released. (2012). IBM SPSS Statistics for Windows, Version 21.0. Armonk, NY: IBM Corp.

KAMIMURA, K. M.; SANTOS, G. R.; OLIVEIRA, M. S.; DIAS JUNIOR, M. SOUZA.; GUIMARÃES, P. T. G. Variabilidade espacial de atributos físicos de um Latossolo Vermelho-Amarelo, sob lavoura cafeeira. Revista Brasileira de Ciência do Solo, v. 37, n. 2, p. 877-88, 2013.

KATO, E., RAMOS, M. L. G., VIEIRA, D. F. A, MEIRA, A. D., MOURÃO, V. C. Propriedades físicas e teor de carbono orgânico de um Latossolo Vermelho-Amarelo do cerrado, sob diferentes coberturas vegetais. Bioscience Journal. v.26, n.4, p.732-738, 2010.

KEMPER, W. D.; CHEPIL, W. S. Size distribution of aggregates. Methods of Soil Analysis: Part 1 Physical and Mineralogical Properties, Including Statistics of Measurement and Sampling, v. 9, p. 499-510, 1965.

LEHMANN, J.; SILVA JÚNIOR, J. P.; STEINER, C.; NEHLS, T.; ZECH, W.; GLASER, B. Nutrient availability and leaching in archaeological anthrosol and a ferralsol of the Central Amazon basin: fertilizer, manure and charcoal amendments. Plant and Soil, v.249, n.2, p. 343-357, 2003.

LOSS, A., BASSO, A., OLIVEIRA, B. S., KOUCHER, L. D. P., OLIVEIRA, R. A. D., KURTZ, C.; COMIN, J. J. Carbono orgânico total e agregação do solo em sistema de plantio direto agroecológico e convencional de cebola. Revista Brasileira de Ciência do Solo, v. 39, n. 4, p. 1212-1224, 2015.

LUCIANO, R. V.; BERTOL, I.; BARBOSA, F. T.; KURTZ, C.; FAYAD, J. A. Propriedades físicas e carbono orgânico do solo sob plantio direto comparados à mata natural, num Cambissolo Háplico. Revista de Ciências Agroveterinárias, v. 9, n. 1, p. 09-19, 2010.

MANTOVANELLI, B. C.; SILVA, D. A. P.; CAMPO, M. C.C.; GOMES, R. P. G.; SOARES, M. D. R.; SANTOS, L. A. C. dos. Avaliação dos atributos do solo sob diferentes usos na região de Humaitá, Amazonas. Revista de Ciências Agrárias, v. 58, n. 2, p. 122-130, 2015. 
MOLINE, E. F. V.; COUTINHO, E. L. M. Atributos químicos de solos da Amazônia Ocidental após sucessão da mata nativa em áreas de cultivo. Revista de Ciências Agrárias, v. 58, n. 1, p. 14-20, 2015.

MOREIRA, A.; MALAVOLTA, E. Dinâmica da matéria orgânica e da biomassa microbiana em solo submetido a diferentes sistemas de manejo na Amazônia Ocidental. Pesquisa Agropecuária Brasileira, v. 39, p. 1103-1110, 2004.

MULLER, M. M. L.; GUIMARÃES, T. D.; MARTINS, P. F. S. Degradação de pastagens na região Amazônica: propriedades físicas do solo e crescimento de raízes. Pesquisa Agropecuária Brasileira, 36:1409-1418, 2001.

NICOLODI, M.; ANGHINONI, I.; GIANELLO, C. Indicadores da acidez do solo para recomendação de calagem no sistema plantio direto. Revista Brasileira Ciência do Solo, v.32, p.237-247, 2008.

Reichert, J.M.; Suzuki, L.E.A.S.; Reinert, D.J.; Compactação de solo em sistemas agropecuários e florestais: identificação, efeitos limites críticos e mitigação. In: Ceretta, C.A.; Silva, L.S.; Reichert, J. M. Tópicos em ciência do solo. Viçosa: Sociedade Brasileira de Ciência do Solo; 2007. vol. 5, p. 49-134.

REIS, M. S.; FERNANDES, A. R.; GRIMALDI, C.; DESJARDINS, T.; GRIMALDI, M. Características químicas dos solos de uma topossequência sob pastagem em uma frente pioneira da Amazônia Oriental. Revista de Ciências Agrárias, n. 52, p. 37-47. 2009.

SANTOS, L. A. C.; CAMPO, M. C. C.; COSTA, H. S.; PEREIRA, A. R. Caracterização de solos em uma topossequência sob terraços aluviais na região do médio rio Madeira (AM). Ambiência, v. 8, n. 2, p. 319-331, 2012.

SCHALLER, F. W.; STOCKINGER, K. R. 1953. A comparison of five methods for expressing aggregation data1. Soil Sci. Soc. Am. Proc. v. 17, n. 4, p. 310-313.

SOUZA, F. G.; CAMPOS, M. C. C.; BRITO FILHO, E. G.; CUNHA, J. M.; LIMA, A. F. L.; SALES, M. C. G.; SANTOS, L. A. C. Physical attributes of soil under amazon forest conversion for different crop systems in southern Amazonas, Brazil. Canadian Journal Soil Science, v.99, p.563-574, 2019.

STRECK, C.A..; REINERT, D.J.; REICHERT, J.M. \& KAISER, D.R. Modificações em propriedades físicas com a compactação do solo causada pelo tráfego induzido de um trator em plantio direto. Ciência Rural, v. 34, n. 3, p. 755-760, 2004.

VIANA, E. T.; BATISTA, M. A.; TORMENA, C. A.; COSTA, A. C. S.; INOUE, T. T. Atributos físicos e carbono orgânico em Latossolo Vermelho sob diferentes sistemas de uso e manejo. Revista Brasileira de Ciência do Solo, v. 35, n.6, p. 2105-2114, 2011. 
WALKLEY, A. BLACK, I. A. An examination of the degtjareff method for determining soil organic matter and a proposed modification of the chromic acid titration method. Soil Science, v. 37, n. 1, p. 29-38, 1934.

WARRICK A. W, NIELSEN DR. Spatial variability of soil physical properties in the field. In: HILLEL D. ed. Applications of soil physics. New York, USA; 1980.

YEOMANS, J. C. BREMNER, J.M. A rapid and precise method for routine determination of organic carbon in soil. Communications in soil science and plant analysis, v. 19, n. 13, p. 1467-1476, 1988.

ZANINETTI, R. A.; MOREIRA, A.; MORAES, L. A. C. Atributos físicos, químicos e biológicos de Latossolo Amarelo na conversão de floresta primária para seringais na Amazônia. Pesquisa Agropecuária Brasileira, v. 51, n. 9, p. 1061-1068, 2016. 